\title{
Tradução e Validação Cultural do Questionário Algofuncional de Lequesne para Osteoartrite de Joelhos e Quadris para a Língua Portuguesa
}

\author{
Translation and Cultural Validation of the Lequesne's \\ Algofunctional Questionnaire for Osteoarthritis of Knee and \\ Hip for Portuguese Language
}

\author{
Felipe C. Marx $^{(1)}$, Leda Magalhães de Oliveira ${ }^{(2)}$, Cintia G. Bellini( ${ }^{(1)}$, Michele Cristina C. Ribeiro $^{(1)}$
}

\section{RESUMO}

Objetivo: tradução, validação e adaptação cultural do índice algofuncional de Lequesne para a língua portuguesa. Pacientes e Métodos: o questionário original, publicado em língua inglesa, foi traduzido por três professores de inglês, e após, retraduzidos por outros três professores. Quatro fisioterapeutas se reuniram e, comparando as traduções, elaboraram a primeira versão. Esta foi aplicada a 11 pacientes com diagnóstico de osteoartrite de joelhos e/ou quadris, e esta versão foi reconhecida plenamente pelos pacientes, que não relataram problemas de compreensão. Passou-se, então, a aplicar esta versão, considerada como definitiva, a outros 73 pacientes da seguinte forma: uma vez pelo observador 1 (Al), no mesmo dia pelo observador 2 (A2) e uma semana mais tarde pelo observador 1 ou 2 (A3). Conjuntamente, colheu-se dados sobre idade e sexo, e aplicou-se o questionário Westerm Ontário and McMaster Universities (WOMAC) em sua versão validada para língua portuguesa. Resultados: para osteoartrite de joelhos, os pacientes do pré-teste ( 10 de 11 avaliados) tinham idade média de 63 anos $(\mathrm{DP}=9,3)$ e dois eram homens. Sua nota média para o índice de Lequesne foi de $14,9(\mathrm{DP}=5,1)$. Os pacientes, avaliados com a versão definitiva, em número de 42 , tinham em média 67,5 anos $(\mathrm{DP}=8,7)$, cinco eram homens. Sua média para o índice de Lequesne foi para $\mathrm{Al}=11,9(\mathrm{DP}=5,0)$, para $\mathrm{A} 2=12,1(\mathrm{DP}=6,4) \mathrm{e}$ $\mathrm{A} 3=11,3(\mathrm{DP}=7,9)$. A correlação intraclasse entre Al e A2 foi de 0,99 e entre Al e A3 foi de 0,99. O coeficiente de Pearson entre Al e WOMAC dor foi de 0,800 , WOMAC rigidez foi de 0,640 e WOMAC função foi 0,828 , todas estatisticamente significantes. Para osteoartrite de quadris, os pacientes do pré-teste ( 3 dos 11 avaliados) tinham idade média de 67 anos (DP=9,18) e todos eram mulheres. Sua nota média para o índice de Lequesne foi de 11,2 $(\mathrm{DP}=5,86)$. Os pacientes, avaliados com a versão definitiva, em número de 37 , tinham em média 66,9 anos (DP=9,01), 8 eram homens. Sua média para o índice de Lequesne foi para $\mathrm{Al}=12,5$

\begin{abstract}
Objective: translation, validation and cultural adaptation of the algofunctional index of Lequesne for the Portuguese language. Patients and Methods: the original questionnaire, published in English, was translated by three English professors, and after back translated by others three professors. Four physiotherapists had congregated and comparing the translations, they had elaborated the first version. This was applied to 11 patients with diagnosis of osteoarthritis (OA) of knees and/or hips, and this version was recognized fully by the patients, who had not reported understanding problems. This version, considered as definitive, was them applied to others 73 patients in the following form: a time for observer 1 (A1), in the same day for observer 2 (A2) and one week later for observer 1 or 2 (A3). Jointly it was collected data on age, sex and applied questionnaire Westerm Ontario and McMaster Universities WOMAC in its version validated for Portuguese language. Results: for osteoarthritis of knees, the patients of the pre-test ( 10 of 11 evaluated) had average age of 63 years $(D P=9.3)$ and 2 were men. Its average grade for the index of Lequesne was of 14.9 (DP=5.1). The patients, evaluated with the definitive version, in number of 42 , had in average 67.5 years $(D P=8.7), 5$ were men. Its average for the index of Lequesne was for $A 1=11.9(D P=5.0)$, for $A 2=12.1(D P=6.4)$ and $A 3=11.3$ $(D P=7.9)$. The intraclass correlation between $A 1$ and $A 2$ was of 0.99 and between $A 1$ and $A 3$ was of 0,99. The Pearson coefficient between $A I$ and WOMAC pain was of 0.800, WOMAC stiffness was of 0.640 and WOMAC function was 0.828 , all with statistical significance. For osteoarthritis of hips, the patients of the pre-test (3 of the 11 evaluated) had average age of 67 years (DP=9.18) and were all women. Its average grade for the index of Lequesne was of $11.2(D P=5.86)$. The patients, evaluated with the definitive version, in number of 37 , had in average 66.9 years $(D P=9.01), 8$ were men. Its average for the index of Lequesne was for $A I=12.5$
\end{abstract}

\footnotetext{
Universidade Federal de São Paulo (UNIFESP). Agradecimento: Giany Gonze Tellini, Mestre em Reabilitação (UNIFESP-2000). Recebido em 13/02/06. Aprovado, após revisão, em 05/07/06.

1. Fisioterapeuta com especialização em Ortopedia pela UNIFESP, em 2003.

2. Fisioterapeuta da Disciplina de Reumatologia da UNIFESP, Mestre em reabilitação (UNIFESP-1994), Professora no Curso de Fisioterapia das Faculdades Metropolitanas Unidas (UniFMU)

Endereço para Correspondência: Felipe C. Marx, Rua Xavier Curado, 285, CEP 04210-100, São Paulo, SP, Brasil, telefone (11) 6215-0981 / 9716 4171, fax: (11) 62156808, e-mail: felipe.marx@uol.com.br
} 
$(\mathrm{DP}=5,6)$, para A2 $=12,5(\mathrm{DP}=5,7)$ e $\mathrm{A} 3=14,1(\mathrm{DP}=6,3)$. A correlação intraclasse entre $\mathrm{Al}$ e $\mathrm{A} 2$ foi de 0,99 e entre $\mathrm{Al}$ e $\mathrm{A} 3$ foi de 0,98. O coeficiente de Pearson entre Al e WOMAC dor foi de 0,759, WOMAC rigidez foi de 0,659 e WOMAC função foi 0,851 , todas também estatisticamente significantes. Conclusão: a versão em língua portuguesa (Brasil) do índice algofuncional de Lequesne, para avaliação de osteoartrite de joelhos e quadris está validada para uso em população brasileira.

Palavras-Chave: osteoartrite, Lequesne, avaliação funcional, osteoartrite de joelho, osteoartrite de quadril.

\section{INTRODUÇÃO}

A osteoartrite é a forma mais comum de doença articular no mundo ocidental. Se caracteriza patologicamente por perda da cartilagem articular e formação marginal osteofitária $^{(1)}$. Embora as causas da osteoartrite sejam ainda mal compreendidas, estresses biomecânicos capazes de atingir a cartilagem articular e osso subcondral, alterações bioquímicas na cartilagem e membrana sinovial, além de fatores genéticos são itens importantes em sua patogênese ${ }^{(2)}$.

Sua prevalência aumenta com a idade, de $7 \%$ entre pessoas de 65-70 anos a 11,2\% entre aqueles com 80 anos ou mais, pouco variando entre os estudos existentes ${ }^{(3,4)}$. Um recente estudo da Organização Mundial da Saúde (OMS) refere que a osteoartrite seria a quarta causa mais importante de incapacidade entre mulheres e a oitava entre homens. Estudos radiográficos mostram algumas alterações em 30\% de homens e mulheres acima de 65 anos, mas apenas um terço destes são sintomáticos ${ }^{(1)}$.

A osteoartrite sintomática progride em um padrão que inclui dor articular, perda de força, incapacidade para marcha e redução da aptidão física ${ }^{(5)}$. Os fatores de risco para o desenvolvimento da osteoartrite incluem obesidade, excesso de esforço articular, lesões periarticulares e alguns riscos ocupacionais ${ }^{(6)}$. Alguns autores consideram também a presença de componentes genéticos ${ }^{(1)}$. Nos membros inferiores (MMII), a osteoartrite tem grande impacto nas articulações de joelhos e quadris. Essa alteração resulta em grande incapacidade para a marcha, transposição de obstáculos (como escadas) e cuidados domésticos ${ }^{(5)}$. Nas mulheres, a osteoartrite de joelhos é mais prevalente, enquanto nos homens a manifestação é mais comum nos quadris $^{(2)}$. Os sintomas mais referidos são dor e perda de função. A incapacidade resultante reduz a qualidade de vida e aumenta riscos de morbidade e mortalidade ${ }^{(1)}$.

Vários consensos foram publicados visando as melhores indicações de tratamento. Embora não haja cura para a osteoartrite, o tratamento é dirigido a cada paciente individualmente, procurando minimizar dor, manter ou
(DP=5.6) for $A 2=12,5(D P=5.7)$ and $A 3=14.1(D P=6.3)$. The intraclass correlation between $A I$ and $A 2$ was of 0.99 and between $A 1$ and $A 3$ was of 0.98 . The coefficient of Pearson between $A I$ and WOMAC pain was of 0.759 , WOMAC rigidity was of 0.659 and WOMAC function was 0.851 , all also with statistical significance. Conclusion: the version for the Portuguese language (Brazil) of the algofunctional index of Lequesne, for evaluation of osteoarthritis of knees and hips is validated for use in Brazilian population.

Keyworks: osteoarthritis, Lequesne, functional evaluation, hip arthrosis, knee arthrosis.

melhorar amplitude de movimento articular e limitar a incapacidade ${ }^{(2,7-9)}$. Entretanto, as decisões terapêuticas dependem da intensidade da dor e do grau de incapacidade física dos pacientes ${ }^{(3,4)}$.

A capacidade funcional dos MMII pode ser avaliada de várias formas, tais como: testes que quantificam restrição de atividade física como caminhada de seis minutos, subida de escadas, suporte de pesos, etc. Outra forma é através do uso de questionários, inquirindo o paciente sobre suas limitações e incapacidades. Este ultimo método é relevante e muito apreciado por sua simplicidade e por avaliar a opinião do paciente sobre suas incapacidades ${ }^{(3,4)}$.

Há vários instrumentos capazes de medir diferentes dimensões do estado de saúde dos pacientes com osteoartrite. Entre estes, há dois extensivamente utilizados. O Westerm Ontario and McMaster Universities (WOMAC), mais empregado nos Estados Unidos e Canadá, pode ser utilizado para avaliar tanto pacientes com osteoartrite de quadril quanto de joelho ${ }^{(10,11)}$. Já o índice de Lequesne possui versões distintas para quadril e para joelho ${ }^{(12)}$.

O questionário de Lequesne foi desenvolvido na França nos anos 70 e publicado pela primeira vez nos anos $80^{(14)}$. Foi atualizado em 1997 e novamente revisado em 2003 por Faucher et $a^{(4)}$. Este índice é composto de 11 questões sobre dor, desconforto e função, sendo seis questões sobre dor e desconforto (sendo uma destas distintas para joelho e outra para quadril), uma sobre distância a caminhar e quatro distintas para quadril ou joelho sobre atividades da vida diária. As pontuações variam de 0 a 24 (sem acometimento a extremamente grave, respectivamente $)^{(3,4,12)}$.

Ambos os instrumentos avaliam sintomas e incapacidade física, mas apenas o índice WOMAC possui validação para uso na população brasileira $^{(13)}$.

\section{OBJETIVO}

Tradução, validação e adaptação cultural do índice algofuncional de Lequesne para a língua portuguesa. 


\section{PACIENTES E MÉTODOS}

\section{O QUESTIONÁRIO DE LEQUESNE}

A versão do questionário de Lequesne utilizada neste trabalho foi a atualizada por Faucher em $2003^{(4)}$ e composto por 11 questões (Quadro 1).

QUADRO 1

Questionário Algofuncional de Lequesne (aplicar separadamente para joelho e Quadril)

\section{Dor ou desconforto}

- Durante o descanso noturno:

- nenhum ou insignificante

- $\quad$ somente em movimento ou em certas posições

- $\quad$ mesmo sem movimento

- $\quad$ rigidez matinal ou dor que diminui após se levantar

- 1 minuto ou menos

- $\quad$ mais de 1 minuto porém menos de 15 minutos

- $\quad$ mais 15 minutos

- depois de andar por 30 minutos

0

1

0 1

2

$0-1$

- $\quad$ enquanto anda

- nenhuma

- $\quad$ somente depois de andar alguma distância

- logo depois de começar a andar e aumenta se continuar a andar

- $\quad$ depois de começar a andar, não aumentando

- $\quad$ ao ficar sentado por muito tempo (2 horas)

- enquanto se levanta da cadeira, sem ajuda dos braços

(somente se quadril) (somente se joelho)

Máxima distância caminhada/andada (pode caminhar com dor):

- $\quad$ sem limite

- $\quad$ mais de $1 \mathrm{~km}$, porém com alguma dificuldade

- aproximadamente $1 \mathrm{~km}$ (em + ou - 15 minutos)

- de 500 a 900 metros (aproximadamente 8 a 15 minutos)

- de 300 a 500 metros

- de 100 a 300 metros

- $\quad$ menos de 100 metros

- com uma bengala ou muleta

- com 2 muletas ou 2 bengalas

Atividades do dia-a-dia/vida diária (Aplicar somente para quadril)*

- colocar as meias inclinando-se para frente

- pegar um objeto no chão

- $\quad$ subir ou descer um andar de escadas

- $\quad$ pode entrar e sair de um carro

$\begin{array}{ll} & 0 \\ & 1 \\ 2 \\ 1 \\ \text { (somente se quadril) } & 0-1 \\ \text { (somente se joelho) } & 0-1\end{array}$

\section{Atividades do dia-a-dia/vida diária (aplicar somente para joelho)*}

- consegue subir um andar de escadas

- consegue descer um andar de escadas

- agachar-se ou ajoelhar-se

- consegue andar em chão irregular / esburacado

$$
\begin{aligned}
& 0-2^{*} \\
& 0-2^{*} \\
& 0-2^{*} \\
& 0-2^{*}
\end{aligned}
$$

\footnotetext{
*Sem dificuldade: 0

Com pouca dificuldade: 0,5

Com dificuldade: 1

Com muita dificuldade: 1,5

Incapaz: 2
} 


\section{MÉTODOS DE TRADUÇÃO}

O método de tradução e validação utilizados seguiu os critérios descritos por Guillemin et a $\left.\right|^{(15)}$.

A tradução do questionário foi realizada por dois tradutores independentes, conhecedores da língua original do questionário e cientes do objetivo do estudo. As duas versões produzidas foram analisadas por uma equipe de profissionais, com a formulação de uma versão única, mantendo as características fundamentais dos conceitos encontrados no questionário original.

\section{BACK TRANSLATION}

Na etapa de back translation, o instrumento produzido na etapa anterior foi traduzido novamente para o idioma original para comparação com o mesmo, por tradutores com conhecimento dos dois idiomas e que, desta vez, não conheciam o objetivo do estudo.

Não sendo constatadas divergências entre as versões original e traduzida, iniciou-se a etapa do pré-teste, a qual consta da aplicação do último instrumento traduzido para língua portuguesa, que manteve as características conceituais do questionário original.

\section{DESCRIÇÃO DA AMOSTRA}

A amostra do pré-teste foi de 11 pacientes selecionados de acordo com os critérios de inclusão e exclusão. Todos os pacientes foram esclarecidos sobre os objetivos da pesquisa, e a participação destes foi vinculada à aceitação e assinatura do termo de consentimento livre e esclarecido.

Os critérios de inclusão foram: os pacientes estavam em atendimento no Lar Escola São Francisco (LESF) ou Hospital São Paulo (HSP). Apenas pacientes alfabetizados (mínimo de três anos) poderiam responder ao questionário. A idade mínima para a inclusão era de 55 anos, não havendo idade máxima.

Os critérios de exclusão foram: pacientes de outras instituições, indivíduos portadores de qualquer outra alteração ortopédica e/ou neurológica que afete os membros inferiores.

Usou-se o método de entrevista, o mesmo de outros vários trabalhos de validação de questionários para o português como $\mathrm{HAQ}^{(16)}, \mathrm{DASH}^{(17)}$, WOMAC ${ }^{(13)}$, entre outros.

Durante a aplicação do questionário do pré-teste, foi acrescida ao questionário uma pergunta referente à compreensão ou não da mesma pelo paciente, baseada numa escala de avaliação da compreensão. A adaptação cultural seria necessária naquelas perguntas classificadas pelos entrevistados como de difícil compreensão, em um número de participantes maior que $10 \%$ da amostra estudada, de acordo com os critérios de Guillemin et a ${ }^{(15)}$.

Juntamente à aplicação do questionário algofuncional de Lequesne, foi também utilizado o instrumento de avaliação WOMAC validado para a língua portuguesa ${ }^{(13)}$, visando avaliar a confiabilidade do questionário traduzido de Lequesne.

Passou-se, então, a aplicar esta versão, considerada como definitiva, a outros 73 pacientes da seguinte forma: uma vez pelo observador $\mathrm{l}$ (Al), no mesmo dia pelo observador 2 (A2) e uma semana mais tarde pelo observador 1 ou 2 (A3). Conjuntamente, colheu-se dados sobre idade e sexo, e aplicou-se o questionário WOMAC em sua versão para língua portuguesa. Após análise estatística e comparativa, pudemos comprovar a confiabilidade do questionário traduzido.

O projeto deste estudo passou pela aprovação do $\mathrm{Co}^{-}$ mitê de Ética em Pesquisa da Universidade Federal de São Paulo (UNIFESP) e está registrado sob o número CEP$1147 / 03$. O autor foi contatado, estando de acordo com a validação de seu questionário para o uso em população brasileira.

\section{ANÁLISE ESTATÍSTICA}

Para análise dos dados demográficos, usou-se a análise descritiva, enquanto para avaliação de correlação entre os dados do instrumento Lequesne com WOMAC usouse a correlação de Pearson e de Spearman. A correlação intraclasse foi utilizada para a avaliação da confiabilidade. Considerou-se estatisticamente significante um $\mathrm{p}<0,05$.

\section{RESULTADOS}

Onze pacientes foram entrevistados para avaliar a compreensão do questionário. Nesta fase foi incluída tanto a avaliação para joelho como para quadril. A única dificuldade de compreensão durante o pré-teste foi identificar as distâncias descritas no questionário (100 metros, 100-300 m, 300$500 \mathrm{~m}, 500-900 \mathrm{~m}, 1 \mathrm{~km}$ ). Como não foram observadas discordâncias significativas, e a compreensão do pré-teste foi acima de $80 \%$, passou-se a considerar esta versão como definitiva, iniciando a fase de testes intra-observadores $\mathrm{e}$ interobservadores.

Os dados demográficos, da amostra do pré-teste, podem ser observados na Tabela 1 . A nota média para o índice de Lequesne para joelho foi de 14,9 (DP=5,1). Para osteoartrite de quadril, a nota média para o índice de Lequesne foi de 11,2 (DP=5,86). 
TABELA 1

DADOS DEMOGRÁFICOS, SEXO, IDADE MÉDIA (Desvio Padrão) dos Pacientes do Pré-Teste e aValiação FINAL DOS PACIENTES ESTUDADOS PARA O QUESTIONÁRIO DE QUADRIL E JOELHO

\begin{tabular}{cc}
\hline $\mathbf{n}$ total & $\mathbf{8 4}$ \\
\hline Sexo da amostra & 13 homens $/ 71$ mulheres \\
\hline Idade pré-teste (quadril) & 67 anos $(\mathrm{DP}=9,1)$ \\
\hline Idade pré-teste (joelho) & 63 anos $(\mathrm{DP}=9,3)$ \\
\hline Idade avaliação final (quadril) & 66,9 anos $(\mathrm{DP}=9,01)$ \\
Idade avaliação final (joelho) & 67,5 anos $(\mathrm{DP}=8,7)$ \\
\hline
\end{tabular}

A amostra para a versão definitiva foi composta de 77 pacientes, dos quais 42 para o questionário de joelho e 35 para o de quadril. A nota média para o índice de Lequesne por avaliador pode ser observada na Tabela 2 . A correlação intraclasse (ICC) entre Al e A2 foi de 0,99 e entre Al e A3 foi de 0,99. O coeficiente de Pearson (PCC) entre Al e WOMAC dor foi de 0,800 . WOMAC rigidez foi de 0,640 e WOMAC função foi 0,828 , todas estatisticamente significantes (Tabela 3 ). Os dados demográficos para os pacientes com osteoartrite de quadril estão dispostos na Tabela 1. A nota média para o índice de Lequesne por avaliador pode ser observada na Tabela 2. O ICC entre $\mathrm{Al}$ e A2 foi de 0,99 e entre Al e A3 foi de 0,98 . O PCC entre $\mathrm{Al}$ e WOMAC dor foi de 0,759 , WOMAC rigidez foi de 0,659 e WOMAC função foi 0,851, todas também estatisticamente significantes (Tabela 3 ).

Durante a fase de avaliação do questionário, obteve-se $92,6 \%$ de compreensão positiva à versão em português do índice de Lequesne.

A grande quantidade de pacientes com osteoartrite classificados como muito grave $(32,22 \%)$ e extremamente grave $(34,44 \%)$, segundo o questionário de Lequesne, deve-se ao fato de muitos dos entrevistados terem sido submetidos aos questionários quando internados para intervenções cirúrgicas no quadril ou joelho, decorrentes da doença em questão. É importante salientar que os dados epidemiológicos desta pesquisa não são demonstrativos da epidemiologia de osteoartrite na população brasileira.

\section{DISCUSSÃO}

Osteoartrite é a doença reumática mais comum, atingindo aproximadamente $30 \%$ dos idosos. Pessoas com osteoartrite sintomática em MMII têm dificuldades crescentes nas atividades funcionais. Quando os joelhos são atingidos, o que ocorre em $10 \%$ dos idosos, a capacidade para subir escadas, levantar de cadeiras, ficar em pé confortavelmente
TABELA 2

DESCRIÇÃO DAS NOTAS MÉDIAS PARA O ÍNDICE DE LEQUESNE PARA JOELHO E QUADRIL, PRÉ-TESTE E AMOSTRA DEFINITIVA. CORRELAÇ̃̃o INTRACLASSE (ICC) ENTRE AVALIADOR 1 E 2 (Al E A2), AVALIADOR 1 E 3 (Al E A3) PARA JOELHO E QUADRIL

\begin{tabular}{c|c|c}
\hline \multicolumn{3}{c}{ Nota média para o indice de Lequesne } \\
\hline & joelho & quadril \\
\hline Pré-teste & $14,9(\mathrm{DP}=5,1)$ & $19,5(\mathrm{DP}=0,7)$ \\
& $\mathrm{A} 1=11,9(\mathrm{DP}=5,0)$ & $\mathrm{A} 1=12,5(\mathrm{DP}=5,6)$ \\
V. Definitiva & $\mathrm{A} 2=12,1(\mathrm{DP}=6,4)$ & $\mathrm{A} 2=12,5(\mathrm{DP}=5,7)$ \\
& $\mathrm{A} 3=11,3(\mathrm{DP}=7,9)$ & $\mathrm{A} 3=14,1(\mathrm{DP}=6,3)$ \\
\hline \multicolumn{3}{c}{$\mathrm{ICC}$} \\
\hline A1 e A2 & joelho & quadril \\
\hline A1 e A3 & $0,99^{* *}$ & $0,99^{* *}$ \\
\hline
\end{tabular}

** $p<0,05$

\section{TABELA 3}

ANÁlise ESTATÍSTICA UTILIZANDO OS COEFICIENTES DE Spearman (ICS) e a correlação de Pearson (ICP), AVALIANDO O QUESTIONÁRIO DE LEQUESNE QUANDO COMPARADO AO ÍNDICE WOMAC NOS DOMÍNIOS DOR, RIGIDEZ E FUNÇÃO

\begin{tabular}{c|cc|c|c|cc}
\hline & \multicolumn{2}{c|}{ dor } & \multicolumn{2}{c}{ rigidez } & \multicolumn{2}{c}{ função } \\
\hline & ICS & ICP & ICS & ICP & ICS & ICP \\
\hline $\begin{array}{c}\text { Lequesne } \\
\text { (Joelho) }\end{array}$ & $0,722^{*}$ & $0,800^{*}$ & $0,564^{*}$ & $0,640^{*}$ & $0,768^{*}$ & $0,828^{*}$ \\
$\begin{array}{c}\text { Lequesne } \\
\text { (Quadril) }\end{array}$ & $0,738^{*}$ & $0,759^{*}$ & $0,623^{*}$ & $0,659^{*}$ & $0,825^{*}$ & $0,851^{*}$ \\
\hline
\end{tabular}

** $p<0,05$

e mesmo andar ficam prejudicadas, resultando em redução da aptidão física, aumento de co-morbidades cardiovasculares e de depressão ${ }^{(3,4,18)}$. A prevalência de osteoartrite de quadril varia de 3 a $11 \%$ nas populações ocidentais com idade acima de 35 anos $^{(19)}$.

A osteoartrite ainda é uma doença com pouca correlação clínica e radiográfica e não há uma boa definição que englobe sintomas disfunção e alterações estruturais. Por isso, questionários que inquiram aspectos de dor, disfunção de marcha e atividades da vida diária (AVD) são preciosos para avaliação do estadiamento da doença e resultados do tratamento ${ }^{(20,21)}$.

Quando se institui um tratamento, deve-se possuir instrumentos capazes de monitorá-lo. No caso da osteoartrite de joelho e quadril, as áreas de maior interesse são as referentes à dor e incapacidade funcional ${ }^{(22,23)}$.

O Outcome Measures in Rheumatology Clinical Trials 
group (OMERACT) recomenda a obrigatoriedade do uso de questões que inquiram sobre dor e função em estudos clínicos, assim como demonstram a importância de questões sobre rigidez ${ }^{(24)}$.

A maioria dos instrumentos utilizados na avaliação de osteoartrite é baseada em questionários. No entanto, não há como aplicar um instrumento de avaliação (questionário) estrangeiro sem realizar a sua validação para o país em que se deseja aplicá-lo, quando se objetiva usá-lo em uma população de cultura e idioma diferentes do país onde o instrumento foi gerado ${ }^{(15,25)}$.

Em 2002, Ludwig et al ${ }^{(26)}$ validaram o questionário de Lequesne para a língua alemã, em sua versão para avaliação de quadril e joelho. Nesse estudo, que comparou os resultados com a aplicação concomitante do WOMAC, a versão alemã do questionário alcançou excelente confiabilidade (88\%), ressaltando ser o índice de avaliação algofuncional de Lequesne um ótimo instrumento para mensuração de dor, capacidade de caminhar e atividades de vida diária para indivíduos portadores de osteoartrite no joelho e quadril. Além disso, este questionário é recomendado internacionalmente pela OMS, para avaliação de osteoartrite, o que também nos influenciou na escolha deste instrumento para validação para a língua portuguesa ${ }^{(26)}$.

Bellamy, visando melhor entendimento das escalas avaliativas, dividiu os grupos articulares em quatro itens: joelho, quadril, mãos, demais articulações. Durante seu estudo, considerou o índice de WOMAC e Lequesne como os mais apropriados para avaliação de osteoartrite de joelho e quadril, tanto em pesquisas com drogas como em tratamentos não-medicamentosos ${ }^{(20)}$. Estes índices começaram a ser recomendados para avaliação em ensaios clínicos em osteoartrite pelo EULAR desde $1985^{(27)}$. A presente pesquisa apresenta bons índices de correlação com WOMAC, sendo 0,722 para Lequesne e WOMAC dor, 0,564 para WOMAC rigidez e 0,768 para WOMAC função.

Ao aplicar o questionário, o índice de compreensão foi classificado como fácil por $92,6 \%$ dos pacientes. Esses dados são superiores aos obtidos na validação deste mesmo questionário para a língua coreana, cuja compreensão ficou em $91 \%$, em um estudo realizado por Bae et al ${ }^{(28)}$. $\mathrm{Na}$ versão alemã, o índice de $88 \%$ foi considerado de excelente confiabilidade por Ludwig et al ${ }^{(26)}$. Os autores do estudo coreano citam o fato de ser difícil encontrar tradutores do questionário habilitados na língua francesa e coreana, o que não ocorreu na tradução e adaptação cultural à língua portuguesa (Brasil), explicado pelo uso da versão inglesa, não havendo dificuldade em se encontrar tradutores habilitados em ambos os idiomas.

A mesma dificuldade na identificação das distâncias descritas no questionário encontradas durante a adaptação cultural, foi encontrada por Bae et a ${ }^{(28)}$ que justificou que a educação coreana não é voltada a esse tipo de conhecimento.

Não encontramos questões que necessitassem de adaptação cultural para a versão brasileira, tampouco verificamos diferenças culturais presentes nas questões propostas no questionário de Lequesne que não se aplicassem ao modo de vida dos pacientes brasileiros. É importante ressaltar que mesmo em culturas diferentes como a francesa, alemã, coreana, árabe e brasileira não houve necessidade de alterações no conteúdo do questionário, sendo que este sempre encontrou boa correlação de confiabilidade e validade em suas aplicações.

Há outros estudos comparativos entre os dois questionários, como o de Bellamy et a ${ }^{(11)}$, que usou o questionário de Lequesne e WOMAC para verificar a superioridade de um medicamento em relação a outro, e verificou serem ambos os questionários estatisticamente eficientes, pois apresentaram resultados semelhantes quanto ao registro da melhora dos pacientes portadores de osteoartrite após o uso da medicação proposta ${ }^{(29)}$. O mesmo fato foi verificado por Pavelká et al ${ }^{30)}$, através de um estudo semelhante, que envolveu outro tipo de medicação. Singh et $a^{(31)} \mathrm{e}$ Berman et al ${ }^{(32)}$ propuseram tratamento para osteoartrite através de acupuntura e observaram a melhora de seus pacientes através das respostas aos questionários de Lequesne e WOMAC, que foram semelhantes ${ }^{(31,32)}$. Acreditamos que, com a validação do questionário de Lequesne no Brasil, trabalhos semelhantes possam ser realizados, pois o instrumento WOMAC foi recentemente validado por Fernandes ${ }^{(13)}$, o que permite que estudos comparativos de propostas de tratamentos sejam avaliadas concomitantemente por dois meios válidos e conceituados mundialmente ${ }^{(13)}$

Eyigor ${ }^{(33)}$ comparando dois tipos de técnicas de fortalecimento em osteoartrite de joelhos encontrou nota média para Lequesne de 10,30 no pré-tratamento, e 6,92 no pós-tratamento, demonstrando melhora nos parâmetros após a aplicação dos tratamentos propostos ${ }^{(33)}$.

Na validação do WOMAC para o português usando o questionário de Lequesne, ainda não validado, obteve-se nota média de 10,33, demonstrando, segundo o autor, boa correlação. As notas médias atingidas durante a validação do instrumento WOMAC foram de 43,21 para dor, 43,45 para rigidez e 37,95 para função ${ }^{(13)}$. 
O trabalho de Faucher ${ }^{(4)}$ avaliando a confiabilidade teste-reteste do questionário de Lequesne conseguiu um ICC de 0,95 para joelho, enquanto no presente estudo, obtivemos 0,99 para joelho e 0,98 para quadril. A presente pesquisa apresenta bons índices de correlação com WOMAC, sendo 0,722 para Lequesne contra WOMAC dor, 0,564 contra WOMAC rigidez e 0,768 contra WOMAC função. Faucher obteve na mesma correlação 0,560 para dor e 0,750 para função contra WOMAC.

$\mathrm{O}$ índice de Lequesne em sua versão árabe (Tunísia) obteve ICC de 0,91 , considerado pelo autor de excelente confiabilidade $^{(34)}$.

\section{REFERÊNCIAS}

1. Jordan KM, Arden NK, Doherty M et al: EULAR Recommendations 2003: an evidence based approach to the management of knee osteoarthritis: Report of a Task Force of the Standing Committee for International Clinical Studies Including Therapeutic Trials (ESCISIT) Ann Rheum Dis 62: 1145-55, 2003.

2. American College of Rheumatology Subcommittee on osteoarthritis Guidelines. Recommendations for the Medical Management of Osteoarthritis of the hip and knee. Arthritis \& Rheum 43: 1905-15, 2000.

3. Faucher M, Poiraudeau S, Levrev-Coleau MM, Rannou F, Fermanian J, Revel M: Algofunctional assessment of knee osteoarthritis: comparison of the test-retest reliability and construct validity of the WOMAC and Lequesne indexes. Osteoarthritis Cartilage 10: 602-10, 2002.

4. Faucher M, Poiraudeau S, Lefevre-Colau MM, Rannou F, Fermanian J, Revel M: Assessment of the test-retest reliability and construct validity of a modified Lequesne index in knee osteoarthritis. Joint Bone Spine 70: 520-5, 2003.

5. Fransen M, Crosbie J, Edmonds J: Phisical Therapy is effective for patients with Osteoarthritis of knee: a Randomized Controlled Clinical Trial. J Rheumatol 28: 156-64, 2003.

6. Dawson J, Juszczak E, Thorogood M, Marks S-A, Dood C, Fitzpatrick R: An investigation of risk factors for symptomatic osteoarthritis of the Knee in women using a life course approach. J Epidemiolol Comunity Health 57: 823-30, 2003.

7. Denoeud L, Mazières B, Payen-Champenois C, Ravaud P: First line treatment of knee osteoarthritis in outpatients in France: adherence to the EULAR 2000 recommendations and factors influencing adherence. Ann Rheumatic Diseases 64: 70-4, 2005.

8. Coimbra IB, Pastor EH, Greve JMD et al: Consenso Brasileiro para o tratamento da osteoartrite (artrose). Rev Bras Reumatol 42: 371-4, 2002.

9. Coimbra IB, Pastor EH, Greve JMD et al: Consenso Brasileiro para o tratamento da osteoartrite (artrose). Rev Bras Reumatol 44: 450-3, 2004.

10. Lequesne M, Mery C, Samson M, Marty M: Comparison between the WOMAC and the Lequesne indices in-patients with knee and hip osteoarthritis. Osteoarthritis Cartilage 6: 441-2, 1998.
Com estes resultados, consideramos estabelecida a confiabilidade e a validade da versão brasileira do índice algofuncional de Lequesne, para joelho e quadril.

\section{CONCLUSÃO}

A versão em língua portuguesa (Brasil) do índice algofuncional de Lequesne, para avaliação de osteoartrite de joelhos e quadris está validada para uso em população brasileira.

Declaramos a inexistência de conflitos de interesse.

11. Bellamy N, Buchaman WW, Goldsmith CH, Campbell J, Stitt LW: Validation study of WOMAC: a health status instrument for measuring clinically important patient relevant outcomes to antirheumatic drug therapy in patients with osteoarthritis of the hip or knee. J Rheumatol 15: 1833-40, 1998.

12. Lequesne MG: The algofunctional indices for hip and knee osteoarthritis. J Rheumatol 779-81, 1997.

13. Fernandes MI: Tradução e validação do questionário de qualidade de vida específico para osteoartrose WOMAC (Western Ontario McMaster Universities) para a língua portuguesa. São Paulo, 2003. 103p. Tese de Mestrado - Universidade Federal de São Paulo - Escola Paulista de Medicina - Reumatologia.

14. Lequesne M, Mery C: European Guidelines for Clinical Trials of new antirheumatics drugs. EULAR Bull 9: 171-5, 1980.

15. Guillemin F, Bombardier C, Beaton D: Cross-cultural adaptation of health-related quality of life measures: literature review and proposed guidelines. J Clin Epidemiol 46: 1417-32, 1993.

16. Ferraz MB, Oliveira LM, Araújo PMP, Atra E: Cross cultural reability of the physical ability dimension of the health assessment questionnaire. J Reumatol 17: 813-7, 1990.

17. Orfale AQ, Araujo PMP, Ferraz MB: Translation into portuguese, Cultural Adaptation and Evaluation of the Reability of the Disabilities of the arm Shoulder and hand questionnaire. Bras J Med Biol Res 38: 293-302, 2005.

18. Fischer NM, Pendergast DR, Gresham GE, Calkins E: Muscle Rehabilitation its effect on Muscular and Functional Performance of Patients with Knee Osteoarthritis. Arch Phys Med Rehabil 72: 367-74, 1991.

19. Zhang W, Doherty M, Arden N et al: EULAR evidence based recommendations for the management of hip osteoarthritis: report of a task force of the EULAR Standing Commitee for International Clinicals Studies Including Therapeutics (ESCISIT). Ann Rheum Dis 64: 669-81, 2005.

20. Bellamy N: Osteoarthritis Clinical Trials: Candidates variables and climetric properties. J Rheumatol 24: 768-78, 1997.

21. Sharma L, Kapoor D, Issa S: Epidemiology of Osteoarthritis: an update Cur op. Rheumatol 18: 147-56, 2006.

22. Roddy E, Zhang W, Doherty N, Arden NK et al: Evidence-based recommendations for the role of exercise in the management of osteoarthritis of the hip and knee - the MOVE consensus. Rheumatology 44: 67-73, 2005.

23. Bellamy N: Pain assessment in osteoarthritis: experience with the 
WOMAC osteoarthritis index. Semin Arthritis and Rheum 18: 14-17, 1989.

24. Weigl M, Cieza A, Harder M et al: Linking osteoarthritis-specific health-status measures to the International Classification of Functioning, Disability, and Health (ICF). Osteoarthritis and Cartilage 11: 519-23, 2003.

25. Lequesne M, Mery C, Samson M, Gerard P: Indexes of severity for osteoarthritis of the hip and knee. Scand J Rheumatol 65(suppl): 85-9, 1987.

26. Ludwing FJ, Melzer CH, Grimming H, Daalmann HH. Cross cultural adaptation of the Lequesne algofunctional indices for German speaking patients with osteoarthritis of the hip and the knee. Rehabilitation 41: 249-57, 2002.

27. Pendleton A, Arden N, Dougados M et al: EULAR Recommendations for the management of knee osteoarthritis: report of a task force of the standing committee for international clinical studies including therapeutic trials (ESCISIT). Annals Rheumatic Diseases 59: 936-44, 2000.

28. Bae SC, Lee HS, Yun HR, Kim TH, Yoo DH, Kim SY: Cross-cultural adaptation and validation of Korean Western Ontario and McMaster Universities (WOMAC) and Lequesne osteoarthritis indices for clinical research. Osteoarthritis Cartilage 9: 746-50, 2001.
29. Bellamy N, Kean WF, Buchaman WW, Gerecz-Simon E, Campbell J. Double blind randomized controlled trial of sodium meclofenamate (Meclomen) and diclofenac sodium (Voltaren): post validation reapplication of the WOMAC Osteoarthritis Index. J Rheumatol 19: 153-9, 1992.

30. Pavelká K, Gatterová J, Olejarová M, Machacek S, Giacovelli G, Rovatti LC. Glucosamine sulfate use and delay of progression of knee osteoarthritis: a 3-year, randomized, placebo-controlled, double blind study. Arch Intern Med 162: 2113-23, 2002.

31. Singh BB, Berman BM, Hadhazy V, Bareta J et al: Clinical decisions in the use of acupuncture as an adjunctive therapy for osteoarthritis of the knee. Altern Ther Health Med 7: 58-65, 2001.

32. Berman BM, Singh BB, Lao L et al: A randomized teal of acupuncture as an adjunctive therapy in osteoarthritis of the knee. Rheumatology 38: 346-54, 1999.

33. Eyigor S: A Comparison fo Muscle Training Methods in Patients with Knee Osteoarthritis. Clin Rheumatol 23: 109-15, 2004.

34. Guermazi M, Mesganni M, Yahia M et al: Traduction en Arabe et édute des qualités métrologiques de l'indice de Lequesne sur une population de gonarthrosiques non-africains - Arab Translation and assessment of metricproperties of the Lequesnes index. Ann Readap Med Phis 47: 195-203, 2004. 